\title{
Mechanical Properties of Laser Beam Melting Components Depending on Various Process Errors
}

\author{
Stefan Kleszczynski ${ }^{1}$, Joschka zur Jacobsmühlen ${ }^{2}$, Jan T. Sehrt ${ }^{1}$, and Gerd Witt ${ }^{1}$ \\ ${ }^{1}$ Institute for Product Engineering, University of Duisburg-Essen, Duisburg, Germany \\ \{stefan.kleszczynski, gerd.witt\} @uni-due.de \\ ${ }^{2}$ Institute of Imaging and Computer Vision, RWTH Aachen University, Aachen, Germany
}

\begin{abstract}
Additive Manufacturing processes are constantly gaining more influence. The layer-wise creation of solid components by joining formless materials allows tool-free generation of parts with very complex geometries. Laser Beam Melting is one possible Additive Manufacturing process which allows the production of metal components with very good mechanical properties suitable for industrial applications. These are for example located in the field of medical technologies or aerospace. Despite this potential a breakthrough of the technology has not occurred yet. One of the main reasons for this issue is the lack of process stability and quality management. Due to the principle of this process, mechanical properties of the components are strongly depending on the process parameters being used for production. As a consequence incorrect parameters or process errors will influence part properties. For that reason possible process errors were identified and documented using high resolution imaging. In a next step tensile test specimens with pre-defined process errors were produced. The influence of these defects on mechanical properties were examined by determining the tensile strength and the elongation at break. The results from mechanical testing are validated with microscopy studies on error samples and tensile specimens. Finally this paper will give a summary of the impact of process errors on mechanical part quality. As an outlook the suitability of high resolution imaging for error detection is discussed. Based on these results a future contribution to quality management is aspired.
\end{abstract}

Keywords: Additive Manufacturing, Laser Beam Melting, process errors, mechanical properties, High Resolution Imaging.

\section{Introduction}

Additive Manufacturing (AM) offers many advantages for manufacturing of complex and individual parts. It provides a tool-free production, whereby physical parts are created from virtual solid models in a layer by layer fashion [1, 2]. In a first step the layer data is gained by slicing virtual 3D CAD models into layers of a certain thickness. Layer information could also be gained by slicing data from 3D scanning or CT scanning. In the following build process the layer information is converted into physical parts by creating and joining the respective layers. The principle of layer creation 
classifies the AM process [3]. Laser Beam Melting (LBM) as an AM process offers the opportunity of small volume production of metal components. Here a thin layer of metal powder is deposited onto the build platform. In a next step the powder is molten into solid material by moving a laser beam (mostly $\mathrm{Nd}$ - or Yb-fibre laser source) across the current cross-section of the part. After this, the build platform is lowered and the two process stages are repeated iteratively until the solid metal part is fully produced (figure 1). As a result of the process the produced components show very good mechanical properties, which are widely comparable to conventionally processed materials $[4,5]$ or in some cases even better [5]. The density of components reaches approximately $100 \%$ [4], [6 - 8]. Potential applications for LBM components are located in the domain of medical implants, FEM optimized lightweight components or the production of turbine blades with internal cooling channels $[1,2,5]$. There are about 158 factors of process influences [9] from which the parameters of laser power, scanning velocity, hatch distance (distance of melt traces) and layer thickness have been reported as the most influencing ones [4, 6, 9]. These main process parameters mentioned are often set into connection by means of the magnitude of volume energy density Ev $[4,6,8]$ which is defined as:

$$
E_{v}=\frac{P_{l}}{h \cdot v_{s} \cdot d}
$$

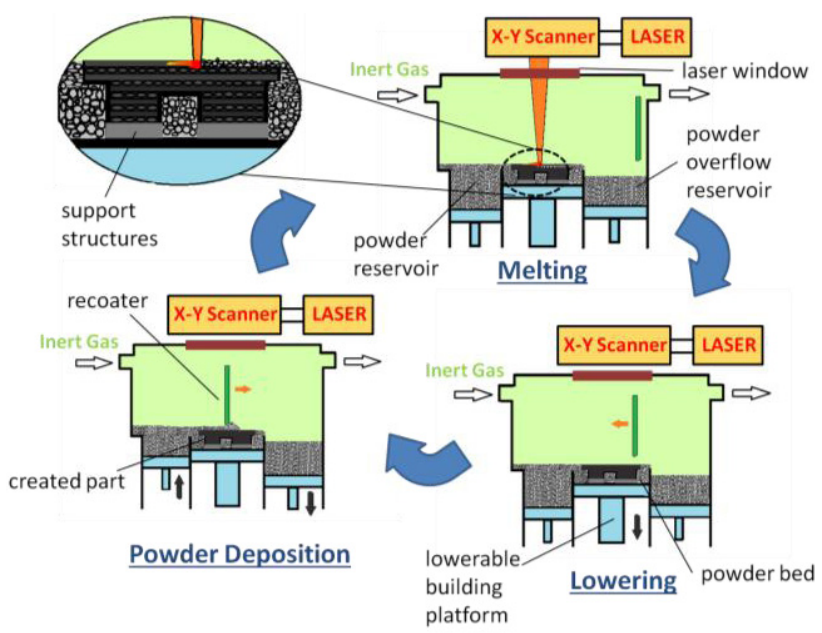

Fig. 1. Schematic process principle of LBM

where Pl stands for Laser Power, $\mathrm{h}$ stands for the hatch distance, v_s stands for the scanning velocity and $d$ stands for the powder layer thickness. Since the process of layer creation determines the resulting part properties $[1,2,4,6]$ wrong process parameters or technical defects in certain machine components could also cause process errors which deteriorate mechanical properties.

Spierings et al. [4] show that the resulting part porosity mainly depends on the used process parameters and significantly affects the mechanical properties. In addition a correlation between volume energy density and the respective part properties is 
investigated with the result that volume energy density can be considered as the parameter determining part porosity. Yasa et al. [7] investigate the influence of double exposure strategies on resulting part properties. It is noted that the application of re-melting is able to improve surface quality and reduce part porosity.

Due to high security requirements of some potential domains of applications and actual standardisation efforts, a demand for suitable quality control for LBM technologies has been reported $[8,10,11]$. Thus far, some different approaches for process control and process monitoring have been given in literature. Kruth et al. monitor the current melt pool using a coaxial imaging system and control laser power to hold the size of the melt pool constant [11]. As the thermal conductivity of metal powder is about three orders of magnitude lower than those of solid metal [6] this system can improve the part quality for overhanging structures by lowering the laser power when the size of the melt pool shows fluctuations in these certain regions. Lott et al. [10] improve this approach by adding additional lighting to resolve melt pool dynamics at higher resolution. In [12] images of the deposited powder layers are taken additionally using a CCD camera. This enables the detection of coating errors due to a damaged coater blade. Doubenskaia et al. [13] use an optical system consisting of an infra-red camera and a pyrometer for visualisation of the build process and online temperature measurements. All approaches previously mentioned feature an implementation into the optical components or the machine housing of the respective LBM system. This makes it elaborate and expensive to equip existing LBM machines with these systems. Moreover the coaxial monitoring systems are limited to the inspection of the melt pool. The result of melting remains uninspected. The CCD camera used in [12] is restricted to the inspection of the powder layer. Possible errors within the compound of melt traces cannot be resolved.

In this work the influence of process errors on resulting part properties is investigated. First selected process errors are provoked and documented using a high resolution imaging system, which is able to detect errors at the scale of single melt traces. A further description of the imaging system is shown in paragraph 2.1 and in $[14,15]$. In general, process errors can influence process stability and part quality [14]. Therefore error samples are built by manipulating the main exposure parameters and exposure strategies. Next, tensile specimens with selected errors are built and tested. The results are validated by microscopy studies on the tested tensile specimens. Finally a correlation between tensile strength, elongation at break, porosity and error type is discussed.

\section{Method}

\subsection{LBM and High Resolution System}

For the experiments in this work an EOSINT M 270 LBM system (EOS GmbH, Germany) is used. Hastelloy X powder is used as material, which is a nickel-base super alloy suitable for applications such as gas turbine blades. The documentation of process errors is carried out with an imaging systems consisting of a monochrome 29 megapixel. CCD camera (SVS29050 by SVS-VISTEK GmbH, Germany). A tilt and 


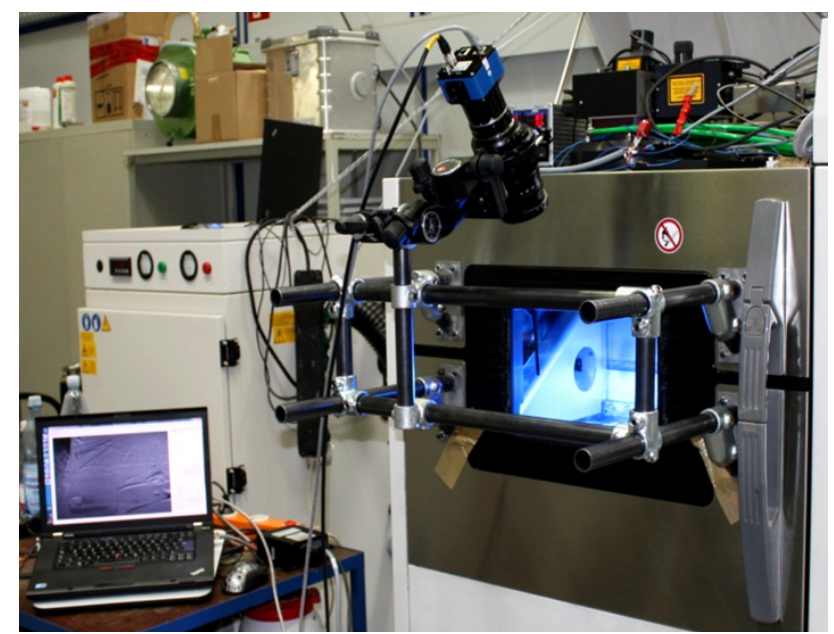

Fig. 2. Camera setup in front of LBM system EOSINT M 270

shift lens (Hartblei Macro 4/120 TS Superrotator by Hartblei, Germany) helps to reduce perspective distortion by shifting the camera back and allows placing the focal plane on the build platform using its tilt ability. A $20 \mathrm{~mm}$ extension tube reduces the minimum object distance of the lens. The imaging system is mounted in front of the LBM system using a tube construction which provides adjustable positioning in height and distance from the machine window (figure 2). Two orthogonally positioned LED line lights provide lighting for the build platform. Matt reflectors on the machine back and the recoater are used to obtain diffuse lighting from a close distance, which was found to yield the best surface images. The field of view is limited to a small substrate platform $(10 \mathrm{~cm} \times 10 \mathrm{~cm})$ to enable best possible resolving power (25 $\mu \mathrm{m} /$ pixel to $35 \mu \mathrm{m} / \mathrm{pixel}$ ) [15]. Image acquisition after powder deposition and laser exposure is triggered automatically using limit switches of the machine's coater blade and laser hourmeter.

\subsection{Determination of Mechanical Properties}

Test specimens for tensile testing are built as cylindrical raw part by LBM. The final specimen shape is produced by milling the raw parts into the standardised shape according to DIN 50125 - B 5x25 [16]. Tensile tests are performed according to the specifications of DIN 50125. A Galdabini Quasar 200 machine is used for the tests. The fragments of test specimens are used for further microscopy studies. For which unstressed material from the specimen's thread heads is prepared into grinding samples. The microscopy studies are carried out using Olympus and Novex microscopes. Porosity of error samples is determined using an optical method according to [7] and [8] where the acquired images are converted to black and white images using a constant threshold value. Finally the ratio of black pixels, representing the porosity, is measured. 


\section{Documentation of Process Errors}

\subsection{Process Errors}

An overview of typical process errors has been given in previous work [14]. In this paper the main focus is on errors that influence part quality and in particularly on errors that affect mechanical properties. As mentioned in paragraph 1 mechanical properties strongly depend on process parameters, which define the energy input for the melting of the powder and in consequence the ratio of distance and width of the single melt traces. Technical defects of the laser source or the choice of wrong process parameter sets could therefore worsen the compound of layers and melt traces leading to porous regions. On the other hand too much energy input could lead to heat accumulation. In this case surface tensions of the melt induce the formation of superelevated regions, which could endanger process stability by causing collisions with the recoating mechanism. However higher energy inputs have been reported to increase mechanical properties due to a better compound of melt traces and lower porosity [7]. To provoke errors of these two kinds the main process parameters laser power, hatch distance and scanning velocity are changed by $20 \%$ and $40 \%$ arround the standard value, which was found by systematic qualification experiments (see [17]). Additionally the layer thickness is doubled from $20 \mu \mathrm{m}$ to $40 \mu \mathrm{m}$ for one sample keeping the process parameters constant. As illustrated in equation 1 these variations directly affect the energy input. Another sample is built using a double exposure strategy resulting in higher energy inputs. For the experiments the stripe exposure strategy is used. Hereby the cross sections of the current parts are separated in stripes of $5 \mathrm{~mm}$ length. The overlap value for these stripes is another process parameter which could affect the compound of melt traces. Therefore one sample with the lowest possible stripe overlap value of $0,01 \mathrm{~mm}$ is build.

\subsection{Error Samples}

Figure 3 shows an image, which was recorded during the build process of error samples using the high resolution imaging system. The samples are arranged in a matrix. The first three rows of the matrix represent the process parameters scanning velocity (vs), laser power $(\mathrm{Pl})$ and hatch distance $(\mathrm{h})$. These values are varied in the columns from $-40 \%$ to $+40 \%$ in steps of $20 \%$.The last row of the matrix contains a reference sample (left), a sample being built with double layer thickness (mid left), a sample being built with double exposure (mid right) and a sample being built with the lowest possible stripe overlap value (right).

As can be seen from figure 3, samples representing higher volume energy densities (reduced scanning velocity/hatch, increased laser power or double exposure) appear much brighter and smoother than those of samples representing low volume energy density (increased scanning velocity/hatch, reduced laser power or double layer thickness). A closer view at the documented error samples (figure 4) shows that surface irregularities and gross particles are visible at the sample of double layer thickness. 


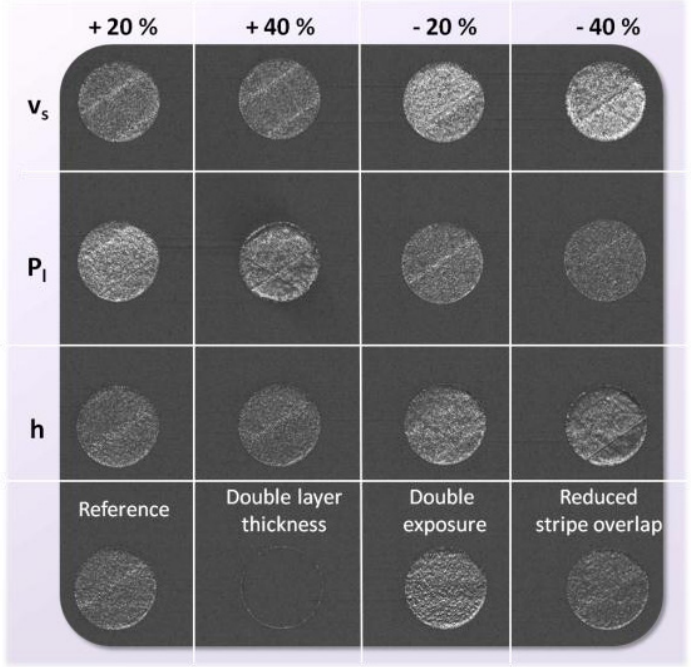

Fig. 3. Documentation of error samples using high resolution imaging
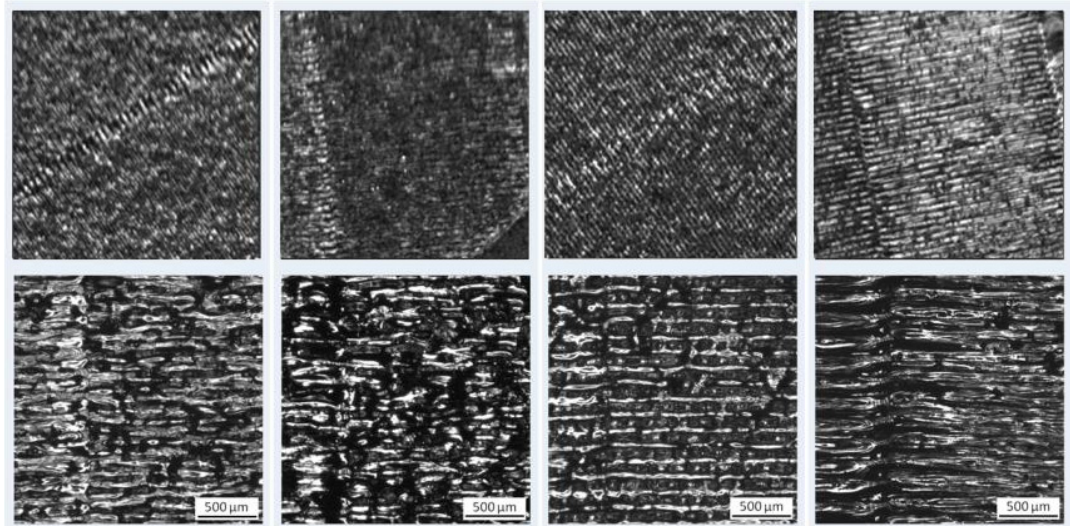

Reference

Double layer thickness

Hatch $+40 \%$

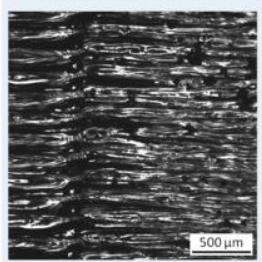

Laser Power $+40 \%$

Fig. 4. Comparison of selected high resolution (top) images vs. images taken from microscopy (bottom)

The sample with $40 \%$ enlarged hatch distance indicates a poor connection of melt traces, which could be a signal for increased porosity. The sample with $40 \%$ increased laser power shows a strong connection of melt traces, although there are some superelevated regions visible at the edges. A comparison of the high resolution images with images taken from microscopy confirms these impressions. Here it is clearly visible that there is no connection between the melt traces at the sample build with enlarged hatch distances. The error samples with $40 \%$ respectively increased or 
decreased parameters show the strongest deviation from the reference sample. These parameter sets are used for production of tensile test specimens. Additionally tensile test specimens representing standard, double layer, double exposure and reduced stripe overlap parameter sets are built.

\section{$4 \quad$ Mechanical Properties}

\subsection{Tensile Strength}

For each type of error six specimens are produced as described in paragraph 2.2 to ensure the level of statistical certainty. The determined values for tensile strength and the associated standard deviations are presented in figure 5. Additionally the calculated values for volume energy density are added into the chart. As can be seen the respective bars representing tensile strength and volume energy density show a similar trend for almost all specimens. In the case of the specimen of "double layer" this trend is not applicable. Higher values for the mean tensile strength are determined (comparing "double layer" to "H $+40 \%$ " and "Vs $+40 \%$ "), although this specimen has the lowest value for the volume energy density. Here it is remarkable that specimen "P - 40 \&" shows a tensile strength which is about $14 \%$ (117 MPa) lower than those of specimen "double layer" while the values for volume energy density are at the same level. Specimens produced using higher energy input parameter sets.

(VS - $40 \%, \mathrm{H}-40 \%, \mathrm{P}+40 \%$, double exposure) exhibit higher values for tensile strength compared to those of the reference specimen. On the other hand specimens produced with low energy input parameters (double layer, $\mathrm{H}+40 \%, \mathrm{Vs}+40 \%$,

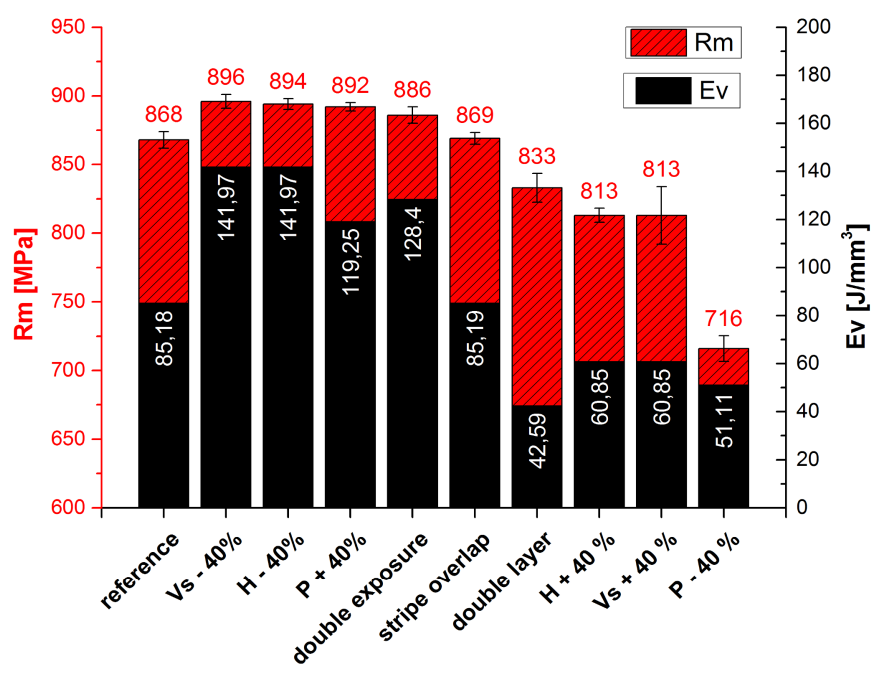

Fig. 5. Results from determination of tensile strength compared to calculated values of volume energy density 
$\mathrm{P}-40 \%$ ) show lower mean values than the reference specimen. Here it is remarkable that the lowest mean value for tensile strength $(716 \mathrm{MPa})$ is determined for specimens built with $40 \%$ decreased laser power. The highest mean value for tensile strength $(896 \mathrm{MPa})$ is achieved at specimens produced with scanning velocities decreased by $40 \%$. The specimens with reduced stripe overlap value showed no significant deviation from reference specimens. Comparing the mean derivations, it is worth mentioning that "high energy" samples show lower values than those of "low energy". Specimens from the type of "Vs $+40 \%$ " show the highest value for mean derivation. The determined mean value for the reference specimens (868 MPa) is comparable to the value determined in previous work $(885 \mathrm{MPa})$ and values from literature $(715$ $\mathrm{MPa}-1110 \mathrm{MPa}$ ) [17]. At this point it has to be stated that the maximum value (1110 $\mathrm{MPa}$ ) is achieved after heat treatment.

\subsection{Elongation at Break}

Figure 6 shows the determined values for the elongation at break compared to the calculated values of volume energy density. Unlike the results for tensile strength, there seems to be no connection between the elongation at break and volume energy density. Furthermore, there are no significant divergent trends recognizable between high energy input and low energy input parameter sets.

It is remarkable that there are three different levels of values recognizable in the chart. First there is the level of about $30 \%$ elongation at break which is determined for most of the specimens (reference, double exposure, stripe overlap, double layer, $\mathrm{Vs}+40 \%)$. Second there is the level of about $25 \%$ to $28 \%$ elongation at break which is detected at four specimens (Vs - $40 \%, \mathrm{H}-40 \%, \mathrm{P}+40 \%, \mathrm{H}+40 \%$ ). Here it is remarkable that the three high energy input parameter sets $(\mathrm{Vs}-40 \%, \mathrm{H}-40 \%$,

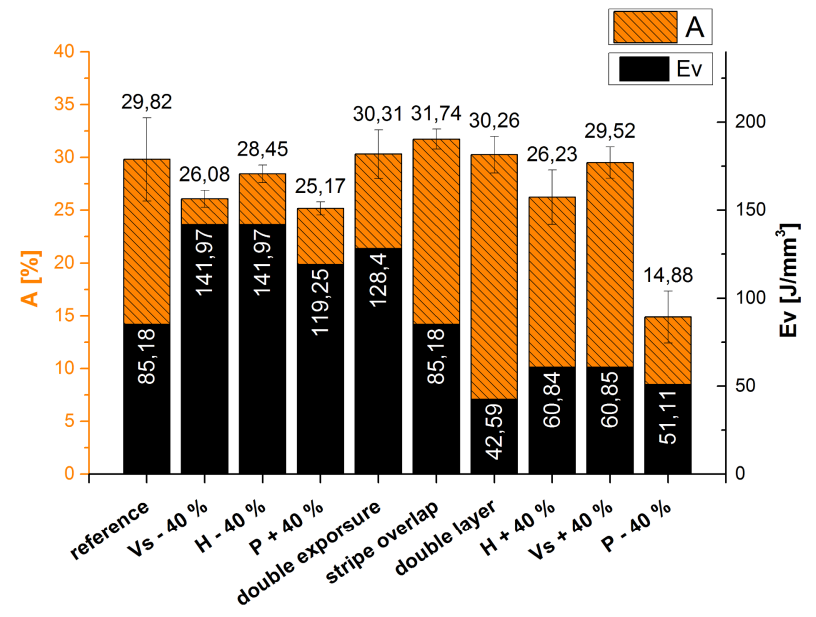

Fig. 6. Results from determination of elongation at break compared to calculated values of volume energy density 
$\mathrm{P}+40 \%)$ show the lowest standard deviation compared to all other specimens. Finally the lowest value for elongation at break is measured for specimen " $\mathrm{P}-40 \%$ " representing the parameter set with lowest energy input. In literature the values for elongation at break for Hastelloy $X$ are located in the range of $22-60 \%$ [17] depending on the respective heat treatment. With exception of specimen "P - $40 \%$ " all determined values are within this range. However the determined values are at least 50 $\%$ lower than the maximal values reported.

\subsection{Porosity}

After mechanical testing, selected specimens are used for determination of porosity using microscopy according to the procedure described in paragraph 2.2. For the reference specimen the porosity is determined to $0,04 \%$, which is comparable to results from previous studies [4 - 7] emphasising that LBM components achieve up to $99 \%$ density. Specimen "P1 - $40 \%$ ", which has the lowest value for volume energy density, shows the highest porosity. The determined value is 3,94\%. The results from porosity analysis (as presented in figure 7) underline previously published statements, which say that porosity grows with sinking energy input. It has to be stated that in general "high energy input" specimens show very similar porosity values $(0,020 \%$ to 0,027 $\%$, see figure 7$)$. The determined porosity values are higher for low energy input specimens $(0,227 \%$ to $3,938 \%)$, which confirms the assumption that porosity is strongly dependent on energy input. The porosity values of the "reference" and the "reduced stripe overlap" specimen differ from each other by $0,02 \%$. Thus the reduced stripe overlap specimens show a lower porosity value. This is remarkable due to the fact that the "reduced stripe overlap" was suspected to increase part porosity. One explanation for this result might be found in the exposure strategy. As mentioned in paragraph 3.1 the cross section of parts are subdivided in stripes of a certain width. After exposure these stripes are rotated and gaps in the compound of melt traces could be closed during exposure of the next layer. On the other hand it has to be stated that the details from photomicrographs used for the analysis show only one certain area of the whole cross section. Moreover pores are distributed stochastically, which makes it difficult to make a statement with an accuracy level of a hundredth of a percent.

\begin{tabular}{ll}
\hline Specimen & Porosity [\%] \\
\hline Reference & 0.04 \\
Vs - 40 \% & 0.025 \\
H - 40 \% & 0.027 \\
Pl + 40 \% & 0.024 \\
Double exposure & 0.025 \\
Reduced stripe overlap & 0.020 \\
Double layer & 0.227 \\
H + 40 \% & 1.633 \\
Vs + 40 \% & 1.176 \\
P - 40 \% & 3.938 \\
\hline
\end{tabular}

Fig. 7. Summary of determined porosity values 


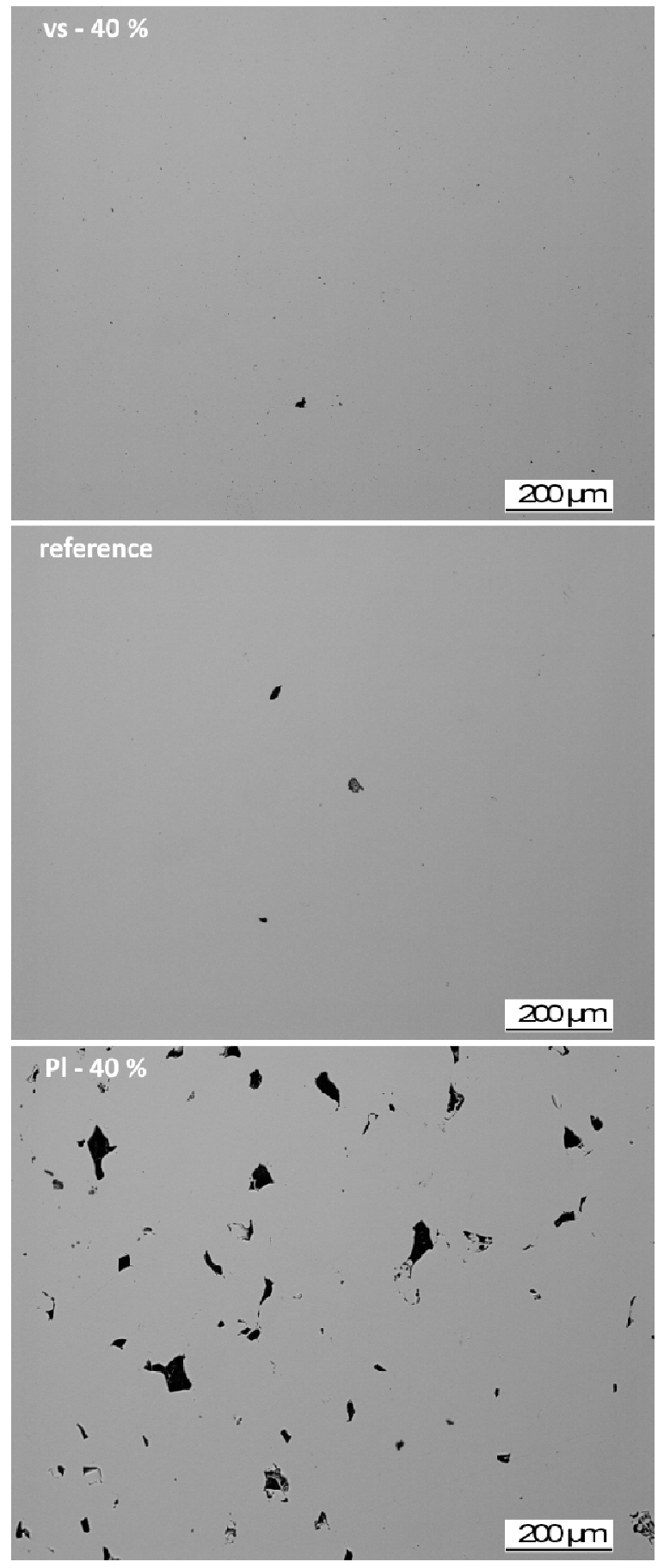

Fig. 8. Photomicrograph from selected test specimens 
Figure 8 shows photomicrographs from reference specimen (middle), reduced scanning speed specimen (top, highest tensile strength) and reduced laser power specimen (at the bottom, lowest tensile strength). Specimen "vs - $40 \%$ " shows little and small pores. The value of porosity is $0,025 \%$. The same appearance is visible at the photomicrographs from the reference sample, which shows slightly more but still small pores. Specimen "Pl - $40 \%$ ", in contrast, shows clearly more and bigger pores, which seem to be distributed stochastically (figure 8 , at the bottom).

\section{Discussion}

The results presented in the previous sections of this paper prove that mechanical properties strongly depend on process parameters. In general it can be stated that increasing energy input improves tensile strength and reduces porosity. It is to be expected that porosity affects tensile strength, due to the fact that irregularities like pores induce crack formation at a certain mechanical load. The elongation at break on the other hand is not systematically affected by different energy input parameter sets. Here, there are some groups of parameter sets which show values at similar levels. However there is no general connection between energy input and the elongation at break for the investigated material. It seems like the exposure strategies have more influence in this case. As can be seen from figure 6 the three high energy input parameter specimens "Vs - $40 \%$ ", "H - $40 \%$ ", "P + $40 \%$ " show similar values for elongation at break. The "double exposure" specimen has a calculated volume energy density which is comparable to those of the other high energy specimens. Nevertheless the elongation at break of this sample lies in the same region as the "reference" sample and some "low energy input" samples. One possible explanation for this appearance could be that the "double exposure" sample was built using two different energy input parameter sets. One for melting the powder and another parameter set for re-melting the produced layer. Thus the heat flow has been different to those of the "high energy input parameter" samples, which has obviously induced different mechanical properties. The "high energy input" specimens show improved values for tensile strength but lower values for elongation at break compared to the reference sample. In contrast the "double exposure" sample shows an improved value for tensile strength at constant ductility.

Figure 9 compares the results of tensile and porosity studies depending on the volume energy density. For this purpose the respective numbers of specimens are plotted into the chart. For identification see the explanation in the chart. Comparing the two plots of logarithmic interpolations shows that both are working contrarily. Both of the magnitudes seem to run asymptotically to parallels of the $\mathrm{x}$-axis for high values of the volume energy density. The tendencies at the tensile tests underline the results from porosity determination (specimen 7: double layer, $\mathrm{Rm}=833 \mathrm{MPa}$, porosity $0.227 \%$, specimen 8: hatch distance plus $40 \%, \mathrm{Rm}=813 \mathrm{MPa}$, porosity $1.633 \%$ ). In this case specimen 8 shows a higher value of porosity and lower tensile strength. Comparing these results with the images from figure 4 allows the conclusion that a poor connection of melt traces causes higher tensile strength values than no connection of melt 
traces. Specimens 7 shows that the previously mentioned correlation between tensile strength, volume energy density and porosity is not applicable to every kind of an error. Here the low value for the volume energy density does not correlate with the interpolation for tensile strength and porosity of other specimens.

This shows that volume energy density is more suitable for estimating tendencies concerning the magnitudes of tensile strength and porosity. A more significant influence is spotted at the type of error, respectively to the kind of energy input or exposure strategy.

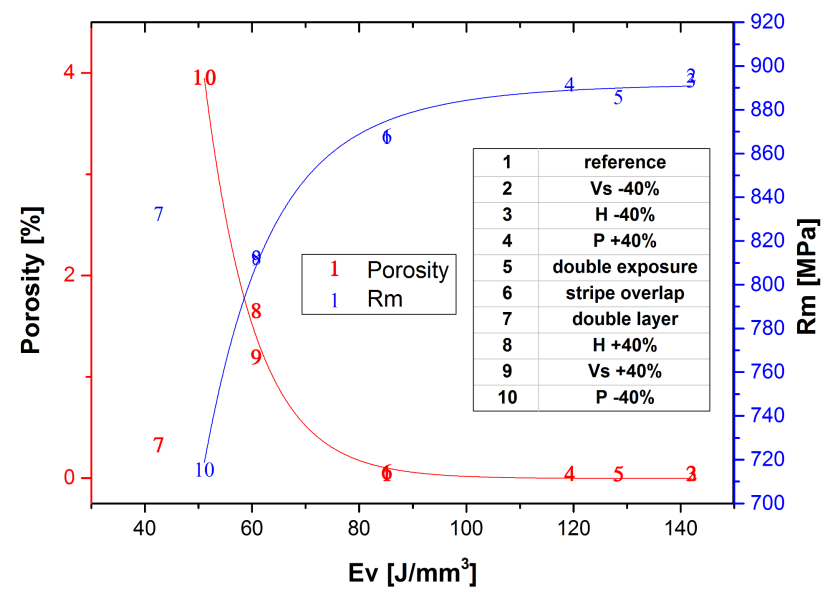

Fig. 9. Connection between porosity, tensile strength and volume energy density

\section{Conclusions}

In this paper a brief demonstration for documenting possible process errors in the area of LBM by using a high resolution imaging system was given. The results and validations via microscopy show a good correlation between the recorded images. High resolution imaging might be an alternative and more pragmatic approach for process monitoring and quality management in the area of LBM due to the fact that the system is easy to implement and compatible to every LBM systems that features a window for the inspection of the process.

In a second step the impact of process errors on tensile strength, porosity and elongation at break was investigated. It could be shown that a higher energy input mostly induces higher values for tensile strength and lower porosities. On the other hand it was found, that the lower the volume energy density is, the lower the determined tensile strength and the higher the porosity are. For some error samples it could be found that the measure of volume energy density is not in a direct correlation with the resulting part properties. This was noticed in detail by comparing the tensile strength of samples with similar values for volume energy density, which were varying for about $117 \mathrm{MPa}$. Here the nature of melt trace connection seems to have the bigger 
influence. The mentioned disagreement of volume energy density with resulting part properties was especially noticed at the determination of the elongation at break. Here some samples that were built with "high energy parameter sets" showed a reduced elongation at break, which induced that the higher energy input seems to embrittle the material compared to the values of the reference specimen. At the same time another specimen, with a comparable higher level of volume energy density resulting in an higher tensile strength, showed higher values for the elongation at break, which were at the same level as specimens produced with low energy input parameters.

Nevertheless all determined mean values for tensile strength and elongation at break were in the range of known values from conventionally produced samples. Only the sample with the lowest tensile strength, lowest elongation at break and highest porosity, which was produced by reducing laser power by $40 \%$, showed values which were at the lower end of the known range. The elongation at break, which is a measure for ductility of materials, did not reach more than $50 \%$ of the known maximum value from literature. This means that for some applications, where high elongation at break values are required, heat treatments are still necessary to improve this certain part property.

For future work the further investigation of the influences of varying process parameters is necessary for different materials and different machine systems, which might use other laser sources or inert gases for flooding of the process chamber. Especially in case of elongation at break it would be interesting to analyse the influence of different exposure strategies. Using high resolution imaging systems for collecting data of different error types and materials could be a useful tool to create a knowledge database, which links process parameters, resulting surface images and resulting mechanical part properties. In a next step an automated image analysis could detect significant differences in the structure of melt traces and might therefore also be applicable to quality management and production documentation.

Acknowledgment. The IGF project $17042 \mathrm{~N}$ initiated by the GFaI (Society for the Promotion of Applied Computer Science, Berlin, Germany), has been funded by the Federal Ministry of Economics and Technology (BMWi) via the German Federation of Industrial Research Associations (AiF) following a decision of the German Bundestag.

\section{References}

1. Gibson, I., Rosen, D.W., Stucker, B.: Additive Manufacturing Technologies - Rapid Prototyping to Direct Digital Manufacturing. Springer, Heidelberg, ISBN 978-1-4419-1119-3

2. Gebhardt, A.: Generative Fertigungsverfahren - Rapid Prototyping - Rapid Tooling - Rapid Manufacturing. Carl Hanser Verlag, München (2007) ISBN: 978-3-446-22666-1

3. N. N.: VDI-Guideline 3404: Additive fabrication - Rapid technologies (rapid prototyping) Fundamentals, terms and definitions, quality parameter, supply agreements. Beuth Verlag, Berlin (2009) 
4. Spierings, A.B., Wegener, K., Levy, G.: Designing material properties locally with Additive Manufacturing technology SLM. In: Proc. of the Solid Freeform Fabrication Symposium, Austin, TX, USA (2012)

5. Wohlers, T.: Wohlers Report 2011 - Annual Worldwide Progress Report, Fort Collins (2011)

6. Meiners, W.: Direktes Selektives Laser Sintern einkomponentiger metallischer Werkstoffe. Ph.D. dissertation, RWTH Aachen University, Aachen, Germany (1999) ISBN: 3-82656571-1

7. Yasa, E., Kruth, J.: Application of Laser Re-Melting on Selective Laser Melting parts. Advances in Production Engineering \& Management 6(4), 259-270 (2011)

8. N. N.: VDI-Guideline 3405 - 2. Entwurf. Additive manufacturing processes, Rapid Manufacturing - Beam melting of metallic parts - Qualification, quality assurance and post processing. Beuth Verlag, Berlin (2012)

9. Sehrt, J.T.: Möglichkeiten und Grenzen bei der generativen Herstellung metallischer Bauteile durch das Strahlschmelzverfahren. Ph.D. dissertation, University of DuisburgEssen, Duisburg, Germany (2010) ISBN: 978-3-8322-9229-4

10. Lott, P., Schleifenbaum, H., Meiners, W., Wissenbach, K., Hinke, C., Bultmann, J.: Design of an Optical system for the in Situ Process Monitoring of Selective Laser Melting (SLM). Physics Procedia 2(pt. A), 683-690 (2011)

11. Kruth, J.-P., Mercelis, P., Van Vaerenbergh, J., Craeghs, T.: Feedback control of selective laser melting. In: Proc. 3rd Int. Conf. on Adv. Research in Virtual and Rapid Prototyping, pp. 521-527 (2007)

12. Craeghs, T., Clijsters, S., Yasa, E., Kruth, J.-P.: Online Quality Control of Selective Laser Melting. In: Proc. of the Solid Freeform Fabrication Symposium, Austin, TX, USA (2011)

13. Doubenskaia, M., Pavlov, M., Chivel, Y.: Optical System for On-Line Monitoring and Temperature Control in Selective Laser Melting Technology. Key Engineering Materials 437, 458-461 (2010)

14. Kleszczynski, S., zur Jacobsmühlen, J., Sehrt, J.T., Witt, G.: Error Detection in Laser Beam Melting Systems by High Resolution Imaging. In: Proc. of the Solid Freeform Fabrication Symposium, Austin, TX, USA (2012)

15. zur Jacobsmühlen, J., Kleszczynski, S., Schneider, D., Sehrt, J.T., Witt, G.: High Resolution Imaging for Inspection of Laser Beam Melting systems. In: Proc. of I2MTC 2013, Minneapolis, MN, USA (2013)

16. N. N.: DIN 50125 - Testing of metallic materials - Tensile test pieces. Beuth Verlag, Berlin (2009)

17. Sehrt, J.T., Witt, G.: Anforderungen an die Qualifizierung neuer Werkstoffe für das Strahlschmelzen. In: Proc. of Seminar: Rapid Manufacturing. IWB Anwenderzentrum Augsburg (July 08, 2010) 